\title{
Comparing Aimed Movements in the Real World and in Virtual Reality
}

\author{
Lei Liu* \\ CWI
}

\author{
Catharina Nieuwenhuizen* \\ Eindhoven University of Technology
}

\author{
Jean-Bernard Martens ${ }^{\S}$ \\ Eindhoven University of Technology
}

\begin{abstract}
The study of aimed movements has a long history, starting at least as far back as 1899 when Woodworth proposed a two-component model in which aimed movements are broken into an initial ballistic phase and an additional control phase. In this paper, we use Woodworth's model for experimentally comparing aimed movements in the real world with those in a virtual environment. Trajectories from real world movements have been collected and compared to trajectories of movements taken from a virtual environment. From this, we show that significant temporal differences arise in both the ballistic and control phases, but the difference is much larger in the control phase; users' improvement is relatively greater in the virtual world than in the real world. They progress more in ballistic phase in the real world, but more in correction phase in the virtual world. These results allow us to better understand the pointing tasks in virtual environments.
\end{abstract}

Index Terms: H5.1 [Information Interfaces and Presentation]: Multimedia Information Systems-Artificial, augmented, and virtual realities; H5.2 [Information Interfaces and Presentation]: User Interfaces-Interaction styles, User-centered design

\section{INTRODUCTION}

Natural interaction in a virtual environment allows users to make use of their intrinsic and well developed skills of how interaction takes place with physical objects in the real world. The quest for providing 'natural interfaces' is an important goal for designing 3D interaction techniques. A key argument is that if 3D interaction techniques can be constructed such that they resemble the physical world, users will be able to optimally apply their everyday skills in manipulating objects.

In this work, we report a study on how users perform a very specific movement in the real world and compare it to how users perform the same movement in a virtual world. For this we have chosen the aimed movement, a basic movement that is found for interaction tasks such as pointing, object selection and tracing. Our goal is not to analyze the machine factors (e.g. stereoscopic viewing, head tracking and tracking latency) which contribute to the efficiency of these movements. Rather, we only aim at identifying if differences occur in the movement and, if so, where and when these differences take place.

Our approach is to record path movements and analytically compare the paths of the movements made during the interaction. In general, there are two ways a movement can be analyzed. First, characteristics of the complete movement, such as total time or displacement, can be introduced. The second approach assumes that the movement consists of several submovements and different submovements provide information about the overall movement. Woodworth's two-component model for aimed movements

\footnotetext{
*e-mail: Lei.Liu@cwi.nl

†e-mail:Robert.van.Liere@cwi.nl

†e-mail:C.J.H.Nieuwenhuizen@tm.tue.nl

§e-mail:J.B.O.S.Martens@tue.nl
}

has been leveraged for this. In 1899, Woodworth proposed that aimed movement consists of an initial, ballistic, impulse phase followed by a current-control phase [8]. The impulse phase is programmed to bring the limb into the vicinity of a target, while the control phase consists of corrective behaviors in which sensory feedback is used to accurately approach the target.

In this study, a controlled experiment where users' path trajectories from real world and virtual world movements have been collected was performed. Path trajectories have been compared using various analysis techniques which allow us to decompose the movements into different submovements. The contributions of this paper are summarized as follows:

- Novel analysis techniques have been developed to decompose trajectories into impulse and control phases.

- From this, we demonstrate that significant temporal differences arise in both the ballistic and control phases, but the difference is much larger in the control phase.

- We also bring out the fact that users improve more in the virtual world than in the real world. The ballistic phase contributes more to the improvement in the real world, but less in the virtual world.

\section{Related Work}

\subsection{Fitts' Law}

Fitts' law, applied to aimed movements, predicts that the time required to move to a target is a function of the distance to the target and the size of the target. Fitts' law is formulated as:

$$
T=a+b \log _{2}\left(\frac{D}{W}+1\right)
$$

where $a$ and $b$ are constants that can be determined experimentally by fitting a straight line to the measured data; $D$ and $W$ are the distance to the target and size of the target, respectively; $T$ is the movement time.

Many VR researchers have used Fitts' law to determine the efficiency of a pointing task under varying factors, such as various viewing and input device tracking conditions $[1,7]$. However, in contrast to our goals, Fitts' law only provides information on the complete movement. Our interest is the analysis of submovements, in the hope that these different submovements provide additional information about the overall movement.

\subsection{Two-Component Model}

Over a century ago, Woodworth proposed a two-component model of rapid aimed movements which suggested that aiming movements are composed of an initial impulse phase and a current control phase [8]. The initial impulse phase, also known as ballistic phase, is programmed under central control to bring the limb into the region of the target, while the current control phase or correction phase comes immediately after the initial impulse phase when the limb enters into the range of the target. It is at this moment that visual feedback is used to generate more small adjustments and corrective behaviors.

In the past century, a number of psychologists have performed experiments following the two-component model, e.g. the 1D [4], $2 \mathrm{D}[3]$ and 3D experiments $[2,5]$. To our knowledge, the twocomponent model has not yet been used in virtual reality for the analysis of aimed movements. 

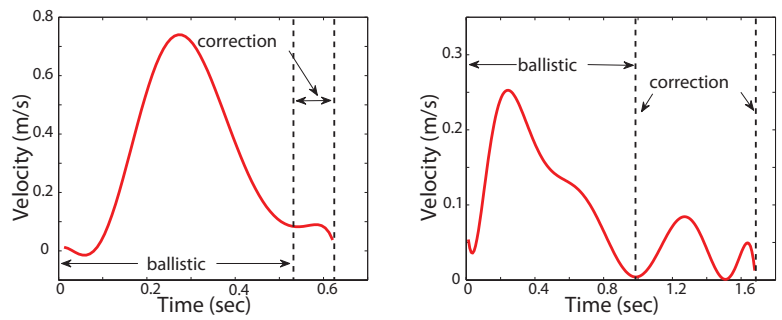

Figure 1: Typical velocity profiles of aimed movements. Left: Real World. Right: Virtual Environment

\subsection{Meyer's criteria on movement parsing}

In order to analyze the complete movement, there must be a way to parse the overall movement into submovements. Parsing criteria have been proposed by Meyer in the stochastic optimizedsubmovement model [4]. To isolate the submovements in 1D, Meyer et al initially smoothed and differentiated the trajectories as a function of time. Then he defined the end of the primary submovement as the first subsequent moment such that either a type 1 , type 2 or type 3 submovement was detected. Type 1 submovement can be interpreted as returning to the target after overshooting. In velocity profile, it occurs when a zero velocity in displacement is reached from positive to negative. Type 2 takes place when a zero acceleration is reached from negative to positive. It corresponds to a local minimum in the velocity profile and often corresponds to undershooting and re-accelerating to the target. Type 3, a slight decrease in the rate of deceleration, is defined as the moment when a zero jerk ${ }^{1}$ is reached from positive to negative.

\section{Movement Parsing}

Although Meyer's criteria allow a more detailed analysis of interaction movements, they were initially designed for 1D movements in the real world. In this section, we introduce new criteria for 3D movements in virtual reality recorded with magnetic trackers.

\subsection{Data filtering}

The recorded position coordinates $(\mathrm{x}, \mathrm{y}, \mathrm{z})$ are filtered as a function of time to avoid spurious details as a result of taking derivatives of noisy signals. A Gaussian filter with a standard deviation of $25 \mathrm{~ms}$ is used to filter the data.

\subsection{Division in ballistic and correction phase}

The division of the movement into its ballistic and correction phase starts by dividing movements into distinct movement intervals, which are separated by pauses. A pause is defined as an interval in between intervals with a zero velocity in which the speed of the cursor remains below 0.05 times the movement's peak speed. For each of the detected movement intervals, it is determined whether or not it makes a considerable contribution to reaching the target. If the path length of a movement interval is more than $25 \%$ of the total path length, it is considered to be part of the ballistic phase. All movement intervals after the ballistic movement intervals are considered to be part of the correction phase.

The detected movement intervals are subsequently divided into submovements. One reason to divide movements into submovements is to use this division for a more detailed description of the movement performance. The other reason is to use this division to determine whether or not the last movement interval of the ballistic phase contains some corrective submovements at the end. Meyer's criteria are adjusted so they could be applied to velocity profiles based on path length:

${ }^{1}$ Jerk is the derivative of acceleration with respect to time

\begin{tabular}{|c|c|c|}
\hline & \multicolumn{1}{|c}{ Meaning } & Math \\
\hline type 1 & overshoot from target & velo $=0$ \\
type 2 & undershoot to target & accel $=0$ \\
type 3 & slowing towards target & jerk $=0$ \\
\hline
\end{tabular}

Table 1: Meyer's parsing criteria

- a type-1 submovement occurs when the speed increases from zero to a value that is above 0.05 times the movement's peak speed (due to the way intervals are defined, this only occurs at the beginning of a movement interval);

- a type-2 submovement occurs at a zero-crossing of acceleration from negative to positive (in combination with a positive jerk that exceeds 0.01 times the maximally observed jerk);

- a type-3 submovement occurs at a zero-crossing of jerk from positive to negative (in combination with a negative value of its derivative that exceeds 0.01 times the maximally observed value).

The thresholds on the slopes of the zero-crossings are applied to avoid the detection of a submovement during small involuntary tremor or slow drift. The minimal requirements for a submovement proposed by Meyer et al were specific for their 1D rotation task and also needed to be adjusted for the 3D interaction task. We chose as the minimal requirements for a submovement that it should traverse a distance of at least $3 \mathrm{~mm}$ and last for at least $75 \mathrm{~ms}$, while the maximum velocity should exceed 0.05 times the maximally observed speed. Submovements that do not meet these requirements are combined with neighboring submovements. This criterion avoids detecting many small, but insignificant, submovements.

The corrective submovements that occur during the last movement interval of the ballistic phase are considered to assist in positioning the pointer within the target area, i.e. they are considered to belong to the correction phase. If the last movement in the ballistic phase consists of multiple submovements, the ballistic phase ends at the first type- 2 submovement that occurs in the last $75 \%-95 \%$ of the traveled path length. Type-3 submovements are only considered to indicate subtle accuracy regulations and are therefore not used to indicate the end of the ballistic phase. If the last ballistic movement interval consists of only one submovement, the end of this movement coincides with the end of the ballistic phase (and the start of the correction phase).

\section{EXPERIMENT}

\subsection{Apparatus}

The experiment was performed in a desktop virtual environment, equipped with a 20-inch viewable stereo-capable Iiyama HA202D DT monitor, a PC with high end GPU, a pair of NuVision 60GX stereoscopic LCD glasses, an ultrasound Logitech 3D head tracker and a Polhemus FASTRAK, used to sample a 6 DOF stylus at $120 \mathrm{~Hz}$. The monitor resolution was $1400^{*} 1050$ at $120 \mathrm{~Hz}$ and the head tracker worked at $60 \mathrm{~Hz}$. The overall end-to-end latency of the FASTRAK during the experiment was measured to be $45 \mathrm{~ms}$, using the method described by Steed [6].

\subsection{Subjects}

The experiment involved 12 skilled computer users among whom 6 were well experienced 3D-virtual-environment users, 6 were 3Dvirtual-reality-naive users. There were 8 right-handed males, 1 lefthanded male and 3 right-handed females.

\subsection{Task}

The experiment was a multi-directional aimed movement task, in which a user first selected a source cylinder and performs an aimed movement to a target cylinder. Twelve target cylinders were arranged around a central source cylinder (see Figure 2). The distance to source varied for each target, but the radius of all cylinders was 
$0.85 \mathrm{~cm}$. The height of the source cylinder was $14 \mathrm{~cm}$, while 6,10 , 14 and $18 \mathrm{~cm}$ for target cylinders.

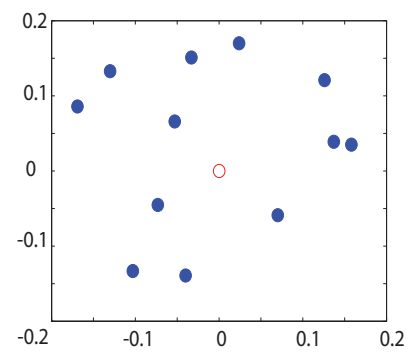

Figure 2: 2D layout of multi-directional aimed movement.(unit:m)

Users needed to press and release the stylus button to start and end a trial. The button-press events must take place in the vicinity of the source or targets, i.e. starting the interaction in a sufficiently close range to the source and ending it within the range of the target.

\subsubsection{Real World Environment}

A physical model, made of a source cylinder and 12 target cylinders, was placed $0.3 \mathrm{~m}$ in front of the users. Users took the tracked stylus to perform the aimed movements (see figure 6, left).

At the start of each trial, the monitor was used to indicate which of the 12 cylinders was the target. ' 0 ' represented the source.

\subsubsection{Virtual Environment}

Users were seated 0.6 meter in front of the CRT monitor. The interaction space was located such that the virtual cylinders were placed at precisely the same location as the real world cylinders were. Users used the same tracked stylus to perform the aimed movements. The visual space was placed 0.3 meter behind the interaction space (see figure 6, right).

To indicate the vicinity of the source and targets, we placed a sphere on top of each cylinder. At the start of each trial, the sphere on the target cylinder was colored red, others remain in blue. When bringing the stylus into the source sphere, users would see a color change from blue to green. If pressing the button of the stylus at that moment, they could start the interaction and the motion would be recorded. Meanwhile, both the source and target spheres would turn to yellow and the color of the background would be altered from grey to black. At the end of the aimed movements, users could find the color changed from yellow to green if they were inside of the target sphere. They needed to press the button to indicate the end. If failed, users would be required to continue until they succeeded.

The differences between the virtual and real world environment were the location of the visual space, the quality of the visual system ${ }^{2}$ and how at the start of each trial the target cylinders were indicated.

\subsection{Procedure}

The trials were grouped into 2 blocks: one block for the real world environment and the other for the virtual environment. A block was composed of 60 trials, 5 repetitions for each of the 12 targets. Trials were given in a random order which, however, was fixed to be the same for a user's real world block and the corresponding virtual world block. A subject could take a break between the trials as well as between blocks.

A practice session in the real world and virtual environment was done before the measurements were taken.

\footnotetext{
${ }^{2}$ Including latency, noise, lower brightness, contrast and frame rate, fewer pixels and polygons rendered per second, etc in VR system.
}

\section{Results}

Before the analysis was done, the data was transformed: log transformations were applied to time, and square root transformations were applied to counts. These transformations resulted that the distribution of the data was closer to the normal distribution assumed by the statistical ANOVA method.

\subsection{Movement parsing}

The movement parsing criteria have been applied on the transformed data. Figure 1 plots the velocity profiles of the ballistic and correction phases as a function of time for one typical trial in real world and one in virtual environment of one user. As can be seen from the figure, there is a difference in the profiles for each phase as well as the total movement. We have also done within-subjects analysis of the data for the overall movement and submovements. The results are provided in Table 2 .

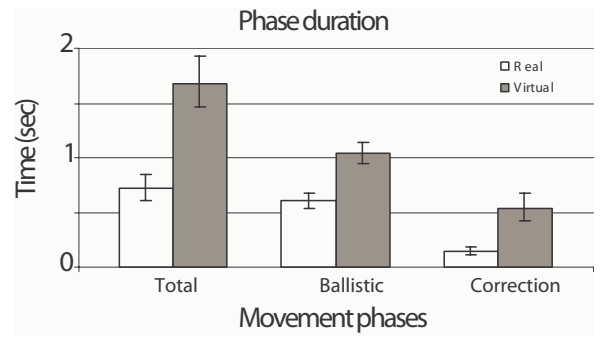

Figure 3: Movement time.

Figure 3 depicts the average duration for the total movement, ballistic and correction phases. It can be seen that there is a significant difference between movement times in the real world and in the virtual world, $(M=0.71, S E=0.03)$ vs $(M=1.68, S E=0.03)$, $F(1,7)=82.17, p<0.0001$. Similarly, it is shown that there is a significant duration difference for both the ballistic and correction phases. Of particular interest is the analysis of the ratio correction / total movement time. For the real world, significantly more time is spent in the ballistic phase than in the correction phase. For the virtual world, it is clear that relatively more time is spent in the correction phase; $(M=20 \%, S E=0.03)$ vs $(M=34.64 \%, S E=0.04)$, $F(1,7)=75.87, p<0.0001$.

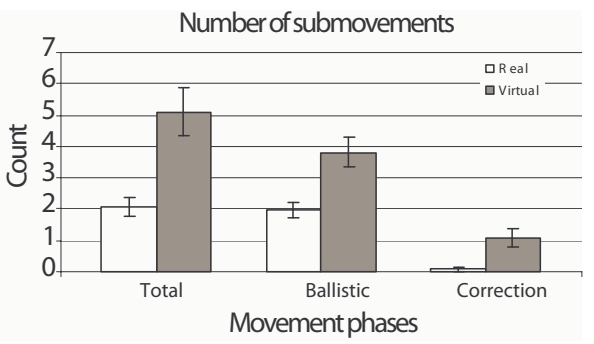

Figure 4: Number of submovements.

A similar analysis can be made for the number of overall submovements and submovements in each phase. As shown in Figure 4, the number of submovements is significantly higher in the virtual world; $(M=2.05, S E=0.04)$ vs $(M=5.03, S E=0.07)$, $F(1,7)=114.24, p<0.0001$.

\subsection{Effect of learning}

Before starting the experiment, the $63 \mathrm{D}$-virtual-reality-naive users were asked to practise 60 trials in both real world and virtual world. The data were also recorded and smoothed by a polynomial curve fitting method. Figure 5 plots the variation of the movement time during the trials in terms of total movement, ballistic and correction phase. The plot demonstrates that the duration of each phase 


\begin{tabular}{|c|cc|cc|c|c|}
\hline & \multicolumn{2}{c|}{ Real World } & \multicolumn{2}{c|}{ Virtual World } & & \\
Measures & Mean & S.E. & Mean & S.E. & F-value & p-value \\
\hline Total Time (s) & 0.71 & 0.03 & 1.68 & 0.03 & 82.17 & .000 \\
Ballistic Phase Time (s) & 0.61 & 0.02 & 1.04 & 0.02 & 76.74 & .000 \\
Correction Phase Time (s) & 0.11 & 0.05 & 0.69 & 0.05 & 111.51 & .000 \\
Proportion of Correction Phase Time & $20.00 \%$ & 0.03 & $34.64 \%$ & 0.04 & 75.87 & .000 \\
Number of Submovements & 2.05 & 0.04 & 5.07 & 0.07 & 114.24 & .000 \\
Number of Submovements Ballistic Phase & 1.97 & 0.04 & 3.80 & 0.05 & 93.88 & .000 \\
Number of Submovements Correction Phase & 0.08 & 0.02 & 1.08 & 0.05 & 84.00 & .000 \\
\hline
\end{tabular}

Table 2: comparing aimed movements in the real world and the virtual reality under various measures.

and total movement decreases sharply within the first 10 trials, but oscillates about an average after the zooming learning period. The reduction for virtual world cases (blue curves) is larger than that of the corresponding real world cases (red curves). The total movement time in the real world drops by $1.68 \mathrm{~s}$ and its ballistic and correction phase are lowered by 0.91 and $0.77 \mathrm{~s}$ on average. In the virtual world, the decreases are $2.26,1.05$ and 1.22 respectively.

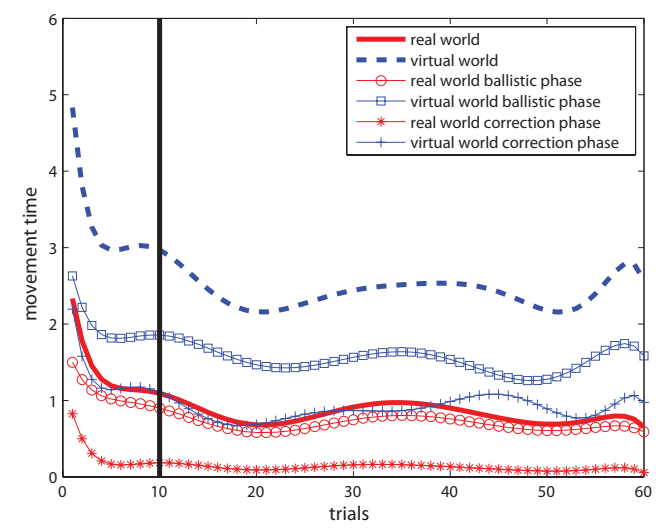

Figure 5: Effect of learning.

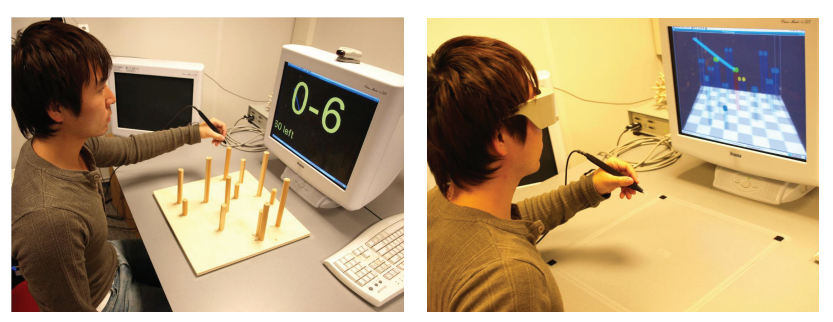

Figure 6: The experimentation platform. Left: Real World Environment. Right: Virtual Environment.

\section{Discussion}

From the movement analysis, we have shown that aimed movements in a virtual environment are less efficient than those in the real world. On average, they take more than two times as long. The ballistic phase is about $70 \%$ longer in the virtual world than in the real world. The greatest difference occurs in the correction phase, where the duration under the virtual environment condition is more than 6 times as long as that in the real world condition. Hence, in a virtual environment, subjects spent significantly more time in the correction phase. According to Woodworth's model, this could be due to either a lower performance of the ballistic movement (larger distance from the target at the end of the ballistic movement) or conflicting visual feedback (lack of depth sensation), which makes performing the correction more difficult and time-consuming.

The analysis of number of submovements also reveals that over $90 \%$ of the trials in the real world have a few submovements, i.e. 1, 2 or 3 . However, in virtual reality, about $75 \%$ of the trials are of 3 to 6 submovements. On the one hand, this specifies that rapid aimed movements in virtual reality are composed of more submovements; on the other hand, due to the large amount of number of submovements, it is clear that the 3D interaction in virtual reality is not fluent and stable, and subjects behave differently among the trials under virtual environment.

Difference also exists in improving the efficiency of the aimed tasks in the real world and virtual reality. As shown by the amount of decreases in movement time, users progress more in the virtual environment than in the real world. This is reasonable because they have already performed well in the real world and the room for improvement is relatively small. Besides, the reduction of the movement time in the real world is more caused by the ballistic phase, which is converse in virtual reality.

\section{Conclusion}

In this paper, we have shown that rapid aimed movements in virtual environments are approximately two times as slow as in the real world. In the ballistic phase, subjects also spent more time in virtual environment, but only 1.7 times as long as in the real world. However, the difference between correction phases is larger. Subjects take on average 6 times as long as they would in the real world to complete the correction movement.

Virtual world aimed movements also differ from real world in the number of submovements needed to complete the task. Real world movements usually contain only a few submovements, whereas in a virtual environment, users perform significantly more submovements. This reveals that the aimed movements are less smooth and stable in a virtual environment.

Besides, users improve more in the virtual world than in the real world. It is the correction phase that contributes more to the improvement in the virtual world, but ballistic phase in the real world.

For future work, we will use our analysis tools to examine how the distance to target and target width in Fitts' law can be integrated with two-component model to automatically facilitate aimed movements.

\section{References}

[1] K. W. Arthur, K. S. Booth, and C. Ware. Evaluating 3d task performance for fish tank virtual worlds. ACM Transactions on Information Systems, 11(3):239-265, july 1993.

[2] L. G. Carlton. Control processes in the production of discrete aiming responses. Journal of Human Movement Studies, 5:115-124, 1988.

[3] R. Chua and D. Elliott. Visual regulation of manual aiming. Human Movement Science, 12:365-401, 1993.

[4] D. E. Meyer, J. E. K. Smith, S. Kornblum, R. A. Abrams, and C. E. Wright. Optimality in human motor performance: ideal control of rapid aimed movements. Psychological Review, 95:340-370, 1988.

[5] A. Murata and H. Iwase. Extending fitts' law to a three-dimensional pointing task. Human Movement Science, 20(6):791-805, Dec. 2001.

[6] A. Steed. A simple method for estimating the latency of interactive, real-time graphics simulations. In Proc. ACM VRST, 2008 [In Press].

[7] C. Ware and R. Balakrishnan. Reaching for objects in vr displays:lag and frame rate. ACM Transactions on Computer-Human Interaction, 1(4):331-356, Dec. 1994.

[8] R. S. Woodworth. The accuracy of voluntary movement. Psychological Review, 3:1-114, 1899. 